\title{
Advanced Two Tier User Authentication Scheme for Heterogeneous Wireless Sensor Networks
}

\author{
Ismail Butun and Ravi Sankar \\ Department of Electrical Engineering, University of South Florida, Tampa, FL, USA \\ e-mails: ibutun@mail.usf.edu, sankar@usf.edu
}

\begin{abstract}
In this paper, we propose a novel User Authentication (UA) scheme for heterogeneous Wireless Sensor Networks (WSNs), which employs both Public Key Cryptography (PKC) and Symmetric Key Cryptography (SKC) approaches, such that it takes advantage of both schemes. Our analysis results have shown that, our scheme is not only more secure and scalable than existing SKC based schemes, but also requires lesser processing power and provides higher energy efficiency than existing PKC based schemes.
\end{abstract}

\section{INTRODUCTION}

For some special applications of WSNs, such as military surveillance, information gathered is sensitive and must be kept confidential. Therefore in such applications UA is necessary. Health care is a good example of UA application for WSNs: Let's say a WSN offers instantaneous medical data service to subscribed health care employees such as doctors and nurses. Since the confidentiality of the data is important (i.e. patient medical records), only the legitimate users should get a response to their queries. Unauthorized users must be prevented from accessing the mentioned confidential information. Therefore UA is a must in these kinds of networks. UA has been well studied for traditional networks, but those solutions cannot be used for WSNs because of the unique characteristics that WSNs possess. UA is an intended feature that will increase data security for WSN users and profitability of the network for WSN providers. In this paper, we propose a secure and scalable UA scheme called Advanced Two Tier User Authentication (ATTUA) to overcome shortcomings of the existing schemes. In our scheme we adopt the idea of a two tier heterogeneous network architecture of [1] in which a user communicates with a sensor node through Cluster Head $(\mathrm{CH})$ of that sensor node. Our scheme uses PKC between $\mathrm{CH}$ s and users, and SKC between $\mathrm{CH}$ s and sensor nodes. In summary, we introduce a UA scheme for heterogeneous WSNs, which inherits most of the advantages of PKC and SKC.

\section{RELATED WORK}

UA for WSNs has been used recently in a few other research papers. Solutions for homogenous WSNs were proposed in [2], [3] and [4]. Wong et al. [2] proposed a dynamic UA scheme for homogenous WSNs. Later this work was improved by Tseng et al. [3] with the following advantages, including resistance of the replay and forgery attacks, reduction of user's password leakage risk, capability of a changeable password, and better efficiency. As discussed in [2], authors claim that weak-password authentication is not suitable for WSNs because it loads the computational overhead to the used cryptography algorithm. In other words the algorithm must be strong enough to compensate for the weakness in the key. Therefore they recommend strong-password authentication for WSNs in which computational load is light owing to the strength in the key. As a result they use SKC throughout the network which is not scalable for a large number of sensor nodes. Although Benenson et al.'s scheme [4] uses PKC, it is not practical for WSNs because of the homogenous network structure, meaning that all the power and processing demanding PKC operations are handled on the normal sensor nodes. As a result authentication operations take minutes and batteries of the sensor nodes deplete faster [5]. To our knowledge, in the literature the only heterogeneous approach to the UA in WSNs is the Le et al.'s [1] Two Tier User Authentication (TTUA) scheme. In the TTUA scheme, $\mathrm{CH}$ s are used as a backbone in the network so that the sensed data, after being collected, are transmitted through $\mathrm{CH}$ s towards the requesting users. Between the $\mathrm{CH}$ s and the users they issue SKC for authentication. It is practically impossible to scale SKC keys to include a large number of users and sensor nodes, because of the memory limitations. Besides, in SKC excluding existing users from the network and including new users to the network, requires key revoking and key re-distribution, which needs a considerable amount of communication overhead. These are the biggest constraints of the TTUA scheme.

\section{AdVAnced Two TIER User AuthentiCATION}

\section{A. System Model:}

In this proposed scheme, WSN consists of basically two elements: 1) $\mathrm{CH}$ s having high processing capability and long lasting power supplies, such as PDAs. 2) Sensor nodes having low processing capability and limited power supplies, such as MICA2 motes. $\mathrm{CH}$ s are assumed as trusted gateways to the sensor nodes. Hence they have better power supplies compared to sensor nodes, they are more convenient to run power hungry PKC algorithms. Therefore between $\mathrm{CH}$ s and users a PKC algorithm, namely ECC, is used for UA purposes. Once a user is authenticated to a $C H$ then allowed to access the sensor nodes through that $C H$. Since it is low power demanding, between $\mathrm{CH}$ s and sensor nodes an SKC algorithm is used. WSN consists of $\mathrm{CHs}$ and sensor nodes, representing a Heterogeneous network structure. ATTUA allows a user to register once and authenticate to the network many times. Users can also change the password anytime at will. We consider wide scale WSN deployed in any variety of environments. In our WSN's architecture, base station $(B S)$ is the point of central control, which serves as a trusted key management facility. $B S$ is many orders of magnitude more powerful than sensor nodes. Typically, $B S \mathrm{~s}$ have enough battery power to surpass the lifetime of all sensor nodes, sufficient memory to store cryptographic keys, stronger processors, and means for communicating with outside networks. After the deployment, sensor nodes form groups, called clusters. For each cluster, a powerful node (e.g. PDA) is assigned as a $\mathrm{CH}$. $\mathrm{CH}$ s have higher communication power than sensor nodes and therefore possess far more radio transmission coverage. $\mathrm{CH}$ can communicate with each other and also with $B S$. In order to protect the keying materials, $\mathrm{CH}$ s are equipped with tamper-resistant hardware. This assumption is reasonable, hence the number of $\mathrm{CH}$ s in a heterogeneous WSN is relatively small (e.g., approximately 20$30 \mathrm{CH}$ s for 1,000 sensors), and the cost of such tamper-resistant hardware is small [6]. Users are equipped with portable computing devices, such as laptops, with no power constraints compared to sensor nodes. Users interact with the WSN for data query and retrieval. After processing sensed information; the sensor node either 
sends the data upon event detection or stores it to serve for the next query.

\section{B. Key Agreement:}

In our scheme we considered a Public Key Infrastructure (PKI) issuing Elliptic Curve Cryptography (ECC) throughout the WSN. The network structure is the same as suggested in [1]. One $B S$ serves as the certification authority for the network. ECC is used for encryption and decryption, Elliptic Curve Digital Signature Algorithm (ECDSA) is used for digital certificate generation and verification. The difference between a digital signature and a certificate is verification of a digital certificate reveals the content whilst verification of a digital signature reveals the hash of the content [7]. Elliptic Curve Diffie Hellman (ECDH) key agreement protocol is used for key agreement between a $C H$ (e.g. $A$ ) and their member sensor node(s), to be used as pair-wise MAC keys, $K_{A, s}$. Initially, $B S$ generates elliptic curve parameters for ECC and ECDSA operations to be used by $B S, C H \mathrm{~s}$ and users, and for ECDH operations to be used by $C H$ s and sensor nodes. These parameters are; base point $P$, private key pri_key $y_{B S}$ and the corresponding public key pub_key $y_{B S}=p r i \_k e y_{B S} \times P$ (where $\times$ stands for elliptic curve point multiplication) itself. $B S$ also generates private-public key pairs for each sensor node $\left(p r i \_k e y_{s}\right.$, $\left.p u b \_k e y_{s}=p r i \_k e y_{s} \times P\right)$ and for each $C H\left(p r i \_k e y_{C H}\right.$, $\left.p u b{ }_{-} k e y_{C H}=p r \bar{i}_{-} k e y_{C H} \times P\right)$. Each sensor is pre-loaded with their private - public key pair and also the public key of the $\mathrm{CH}$ s. Each $C H$ is pre-loaded with their private-public key pair and also the public keys of the sensor nodes.

1) Key agreement between $\mathrm{CH}$ s and sensor nodes: Our scheme uses ECDH key agreement protocol as discussed in [8], between $\mathrm{CH}$ s and sensor nodes in order to let both parties agree on a shared secret key. ECDH allows two parties to agree on the secret key of the SKC algorithm they are using: in our case it is MAC. As discussed in 'Key Agreement' section, in order to reduce energy consumption, all public keys needed for $\mathrm{ECDH}$ protocol are exchanged between sensor nodes and corresponding $\mathrm{CH}$ s before the deployment. Thus, no further communication is needed to exchange public keys. After deployment, each sensor node $(s)$ computes a shared secret key $\left(K_{A, s}\right)$ with its $C H$ (e.g. $A$ ), for authentication purposes as follows:

$s$ computes the elliptic point $R_{s}$ :

$R_{s}=\left(x_{s}, y_{s}\right)=$ pri_key $_{s} \times p u b \_k e y_{A}$

$A$ also computes another elliptic point $R_{A}$ :

$$
R_{A}=\left(x_{A}, y_{A}\right)=\text { pri_key }_{A} \times p u b \_k e y_{s}
$$

Since pri_key $_{s} \times p u b_{-} k e y_{A}=$ pri_key $_{s} \times$ pri_key $_{A} \times P=$ pri_key $A \times p r i \_k e y_{s} \times P=p r i \_k e y_{A} \times p u b \_k e y_{s}, R_{s}=R_{A}$, so does $x_{s}=x_{A}$. As a result $K_{A, s}=x_{s}$ is assigned as the shared secret key between $s$ and $A$.

\section{Authentication:}

ATTUA includes three phases: Registration, Authentication, and Password Change.

1) User registration: User sends a request with his ID encrypted with the public key of the $B S$ (encrypt $t_{p u b \_k e y_{B}}\left(I D_{U}\right)$ ) to the $B S$ for registration to the WSN. $B S$ has the ID list of the legitimate users and provides each legitimate user a certificate. $B S$ has private and public key pair (pri_key $\left.y_{B S}, p u b \_k e y_{B S}\right)$ and the certificate is the user's $I D$ signed by the $B S$, using the private key $\left(p r i \_k e y_{B S}\right)$. $B S$ sends back the certificate to the user. In user authentication phase, with the public key of the $B S\left(p u b \_k e y_{B S}\right)$, each $C H$ can verify the certificate of the user and extract the $I D$ of the user, namely $I D_{U}$.

2) User authentication: All the communications within the network are routed by the $C H$ s. Let us consider the scenario where the user wants to access data aggregated at a sensor $s$ (suppose $A$ is $C H$ of $s$ ), and let us also assume that $A$ is the closest $C H$ in the proximity of the user (intra communications and authentications among $\mathrm{CH}$ are beyond the scope of our paper). Then the authentication process includes the following steps:

Step 1) The user sends his certificate $\operatorname{cert}_{U}$ and time stamp $T_{U}$ along with the hash value of those concatenated by user ID, $I D_{U}$ to $A: \quad$ user $\rightarrow A:$ cert $_{U}, T_{U}, H\left(\operatorname{cert}_{U}\left\|T_{U}\right\| I D_{U}\right)$, where $\|$ means concatenation and $\mathrm{H}$ stands for hashing algorithm such as SHA-1. Upon receiving an authentication request from the user, $A$ first checks whether $T_{U}$ is valid, if yes then it can verify the certificate of the user by using the public key of the $B S\left(p u b \_k e y_{B S}\right)$ and extract the $I D$ of the user, namely $I D_{U}$. Finally $A$ verifies the hash value of the user by using the ID of the user.

Step 2) If the verification is successful, $A$ sends $s$, users' identification $\left(I D_{U}\right)$, its identification $\left(I D_{A}\right)$ and time stamp $\left(T_{A}\right)$ along with a MAC using its shared pair-wise key $\left(K_{A, s}\right)$ with the sensor $s$, $M A C\left(K_{A, s}, I D_{U}\left\|I D_{A}\right\| T_{A}\right)$. Upon receiving the message, $s$ first checks if $T_{A}$ is valid. If yes, it verifies $I D_{U}$ and $I D_{A}$ by generating a MAC with the shared pair-wise key with $A\left(K_{A, s}\right)$ and comparing it with the received MAC. If all of these are successful, then the user is authentic. After successful authentication, sensor $s$ is ready to send data to the user. $s$ may send a short message to inform the user that he is authenticated via $A$.

3) Password change: ATTUA also allows users to change their password at will. Users can do so through $B S$. The user encrypts the new public key $\left(p u b \_k e y_{U}^{*}\right)$ and its new ID $I D_{U}^{*}$ with its current public key $\left(p u b \_k e y_{U}\right)$. After receiving the encrypted message, $B S$ decrypts it by using the current public key of the user (pub_keyU). Then $B S$ can sign the new ID $\left(I D_{U}^{*}\right)$ by its private key $\left(p r i \_k e y_{B S}\right)$ to obtain a new certificate $\left(\operatorname{cert}_{U}^{*}\right)$ and send it back to the user.

\section{SECURITY ANALYSIS}

In this section, we analyze the security of the ATTUA scheme. With the recent progress in ECC, Wang et al. [9] shows that PKC can be more advantageous than SKC not only with key management but also in terms of the memory usage and security resilience. This is another reason to adopt PKC to our ATTUA scheme. In ATTUA users can be added and revoked on the fly. $\mathrm{CH}$ s only need to keep the public key of the $B S$. Whereas in TTUA $C H$ s need to keep the password list of the users every time. In the TTUA scheme, the hash value of the user password is sent to the $B S$ through a secure channel. Also, the hash values list of the $C H$ s secret keys is sent from $B S$ to the user through the secure channel. It means that in case of any intrusion into the secure channel, the WSN would be compromised. In our ATTUA scheme, owing to the Public Key Infrastructure (PKI), a secure channel is not needed between user and $B S$. Therefore users do not have to plug in to the $B S$ for key exchange. This means that users in our network are free to move anywhere but the coverage area of the $B S$. In TTUA if the secret key $\left(K_{A}\right)$ of the $C H A$ is captured, then the network is compromised and all the user passwords stored on $A$ must be revoked. In TTUA scheme, users change their passwords through $\mathrm{CH}$ s. In our ATTUA scheme, users directly communicate with $B S$ to change the password. Since $C H$ is not involved in the password change session, ATTUA is less vulnerable compared to TTUA. In TTUA scheme, a hash function (SHA-1) is used to secure the authentication message between the user and the $C H$. In our ATTUA scheme, we use ECC which is more secure than SHA-1 given that both use same sized keys.

\section{A. Node compromising attacks}

Since $\mathrm{CH}$ s are equipped with tamper resistant hardware, it is impossible to compromise them. This way the SKC pair-wise keys between each sensor and associated $\mathrm{CH}$ is secured on the $\mathrm{CH}$ side. Also the PKC keying materials between $\mathrm{CH}$ s and users are also secured. The weakest element of our proposed scheme is the sensor nodes, since they do not have tamper resistant hardware. In terms of security, we do not let sensor nodes carry any valuable information to compromise the overall WSN. Hence the secret keys between a $\mathrm{CH}$ and each member sensor node are different, the furthest point any attacker can reach is the compromising of the link communication 
between the sensor node captured and the related $\mathrm{CH}$. To defend this, in our ATTUA scheme the secret keys between $\mathrm{CH}$ s and sensor nodes are updated at certain periods with ECDH protocol.

\section{B. Replay attacks}

In the ATTUA scheme an attacker cannot re-use the previous successful login message $H\left(\operatorname{cert}_{U}\left\|T_{U}\right\| I D_{U}\right)$, because the time stamp $T_{U}$ generated by the user protects this message to be used again after a certain time. After the useful time passes, $\mathrm{CH}$ will not allow access to the user. Thus, reply attacks are defended that way.

\section{Impersonation attacks}

Our proposed scheme is resilient against impersonation attacks in the following manner: In authentication phase, an outsider tries to impersonate the login message $H\left(\operatorname{cert}_{U}\left\|T_{U}\right\| I D_{U}\right)$ by fabricating $I D_{U}$ as $I D_{U-\text { guessed. }}$ If outsiders do not know the public key of the $B S$, they cannot verify $\operatorname{cert}_{U}$ and extract $I D_{U}$. Therefore the fabricated ID will change the hash value and will be caught by the $\mathrm{CH}$ :

$$
H\left(\operatorname{cert}_{U}\left\|T_{U}\right\| I D_{U-\text { guessed }}\right) !=H\left(\operatorname{cert}_{U}\left\|T_{U}\right\| I D_{U}\right)
$$

\section{PERformance Evaluation}

In this section we analytically evaluate the performance of our proposed ATTUA scheme and compare it to the TTUA scheme for the following criterions: storage requirement (memory), scalability and communication overhead.

\section{A. Storage}

The TTUA scheme requires each $C H$ to store user $I D$ s and hashed password values, which adds up with the increasing number of users. As mentioned in [1], for the TTUA scheme, assuming that there are $n$ number of users, user $I D$ size is 8 bytes, and the hashed password value is 20 bytes, each $C H$ has to store $n \times 28$ bytes of data for the users. Whereas in the ATTUA scheme, to authenticate the users, the only key that $C H \mathrm{~s}$ have to store in their memory is the public key of the $B S$ (pub_keyBS). This advantage is brought by the PKC. In our scheme, since we use 160 bit (20 bytes) elliptic curves, the public key size is 40 bytes (keep in mind that, for a 160 bit elliptic curve, certificate is 40 bytes long, public key is 40 bytes long and private key is 20 bytes long). Assuming $n=10,000$ the memory required is 40 bytes for ATTUA scheme and 280,000 bytes for TTUA scheme.

\section{B. Scalability}

As mentioned in the previous section, owing to the PKC approach, the memory space available on $\mathrm{CH}$ s in ATTUA scheme does not change with the number of users. So we can state that there is no limit on the number of users. Literally speaking, ATTUA scheme may manage thousands of users without any problem.

Where as in TTUA scheme, memory space available on $\mathrm{CH}$ s is inversely proportional to the number of users. If we want to limit the memory space used to store the keys of users, this will limit the total number of users accordingly. Assume that this space is allocated as 2,800 bytes. This will allow us $(28 \mathrm{x} n=2,800)$ to register up to 100 users to the network.

Here I can state that ATTUA scheme is much more scalable in terms of number of network users.

\section{Communication}

For communication cost, we are interested in the communications involving either $\mathrm{CH}$ s or sensor nodes. To calculate communications cost, we define a number of notations as follows (all of these are in number of hops):

- $C_{b r}$ : Communication cost for broadcasting user ID and password to all $\mathrm{CH}$ s
TABLE I

COMPARISON OF COMMUNICATION COST

\begin{tabular}{|c|c|c|}
\hline Phase & ATTUA & TTUA \\
\hline Registration & 0 & $C_{b r}$ \\
\hline Authentication & $2 C_{U-A}+2 C_{A-s}$ & $2 C_{U-A}+2 C_{A-s}$ \\
\hline Total & $2 C_{U-A}+2 C_{A-s}$ & $C_{b r}+2 C_{U-A}+2 C_{A-s}$ \\
\hline
\end{tabular}

- $C_{U-A}$ : Communication cost between the user and the cluster head $A$

- $C_{A-s}$ : Communication cost between $A$ and $s$

For registration phase, ATTUA has no cost on $\mathrm{CH}$ s or sensor nodes, whereas TTUA needs to broadcast user ID's and passwords to all $\mathrm{CH}$ s. For authentication phase, both schemes have the same cost, 2 messages sent between user and $C H$, and 2 messages sent between $\mathrm{CH}$ and sensor node. The communication costs are summarized in Table I. So as a summary, our ATTUA scheme outperforms TTUA scheme in terms of communications overhead.

\section{CONClusion And Future Work}

In this paper we propose a novel UA scheme for heterogeneous WSNs, called Advanced Two Tier User Authentication scheme (ATTUA), which employs both the PKC and SKC approaches such that it takes advantage of both schemes. Our analysis results have shown that, it is not only more secure and scalable than existing SKC based schemes, but also requires less processing power and provides higher energy efficiency than existing PKC based schemes. In our future work, not only the software simulation (on ns-2 [10], or etc.) but also hardware implementation (with our real sensor devices, namely MICA2 motes) of the proposed ATTUA scheme will be carried out and the resulting outcomes will be published.

\section{REFERENCES}

[1] X.H. Le, S. Lee, and Y.K. Lee. "Two-Tier User Authentication Scheme for Heterogeneous Sensor Networks." the 5th IEEE International Conference on Distributed Computing in Sensor Systems, (DCOSS '09), Marina Del Rey, California, USA, June 8-10, 2009.

[2] K.H.M. Wong, Y. Zheng, J. Cao, and S. Wang. "A dynamic user authentication scheme for wireless sensor networks." IEEE International Conference on Sensor Networks, Ubiquitous, and Trustworthy Computing, 2006.

[3] H.R. Tseng, R.H. Jan, and W. Yang. "An Improved Dynamic User Authentication Scheme for Wireless Sensor Networks." IEEE Global Communications Conference, (GLOBECOM 2007), USA, November 2007, pp. 986-990.

[4] Z. Benenson, N. Gedicke, and O. Raivio, "Realizing robust user authentication in sensor networks." in Worshop on Real-World Wireless Sensor Networks, 2005.

[5] A.S. Wander, N. Gura, H. Eberle, V. Gupta, and S.C. Shantz. "Energy analysis of public-key cryptography for wireless sensor networks." in Proceedings of the Third IEEE International Conference on Pervasive Computing and Communications, 2005, pp. 324-328.

[6] X. Du, G. Mohsen, X.O. Yang, C. Hsiao-Hwa. "Two Tier Secure Routing Protocol for Heterogeneous Sensor Networks." IEEE Transactions on Wireless Communications, Vol.6 (9), September 2007, pp. 3395-3401.

[7] A. Aziz and W. Diffie, "Privacy and authentication for wireless local area networks.” IEEE Personal Communications Magazine, 1(1):25-31, 1994.

[8] X.H. Le, S. Lee, I. Butun, M. Khalid, and R. Sankar, "An EnergyEfficient Access Control Scheme for Wireless Sensor Networks based on Elliptic Curve Cryptography." Journal of Communications and Networks, vol. 5, no. 3, 2009.

[9] H.Wang, B. Sheng, C. C. Tan, and Q. Li, "Comparing symmetric-key and public-key based security schemes in sensor networks: A case study of user access control." in 28th International Conference on Distributed Computing Systems, ICDCS'08, 2008, pp. 11-18.

[10] NS-2 - Network Simulator and Emulator, http://www.isi.edu/nsnam/ns 